\title{
A method for semi-automatic explicitation of agent's behavior: application to the study of an immersive driving simulator
}

\author{
Kévin Darty ${ }^{1}$, Julien Saunier ${ }^{2}$ and Nicolas Sabouret ${ }^{3}$ \\ ${ }^{1}$ Laboratory for Road Operations, Perception, Simulators and Simulations (LEPSIS), French Institute of Science and \\ Technology for Transport, Development and Networks (IFSTTAR), 14-20 Boulevard Newton Cité Descartes, 77447 Marne \\ la Vallée - France \\ ${ }^{2}$ Computer Science, Information Processing and Systems Laboratory (LITIS), National Institute of Applied Sciences (INSA) \\ of Rouen, Avenue de l'Université - BP8, 76801 Saint-Étienne-du-Rouvray Cedex - France \\ ${ }^{3}$ Computer Sciences Laboratory for Mechanics and Engineering Sciences (LIMSI), National Center for Scientific Research \\ (CNRS), BP133, 91403 Orsay Cedex - France \\ kevin.darty@ifsttar.fr, julien.saunier@insa-rouen.fr, nicolas.sabouret@limsi.fr
}

\begin{abstract}
Keywords: Multi-agent simulation, credibility evaluation, objective and subjective approach, behavior clustering and explicitation

Abstract: This paper presents a method for evaluating the credibility of agents' behaviors in immersive multi-agent simulations. It combines two approaches. The first one is based on a qualitative analysis of questionnaires filled by the users and annotations filled by others participants to draw categories of users (related to their behavior in the context of the simulation or in real life). The second one carries out a quantitative behavior data collection during simulations in order to automatically extract behavior clusters. We then study the similarities between user categories, participants' annotations and behavior clusters. Afterward, relying on user categories and annotations, we compare human behaviors to agent ones in order to evaluate the agents' credibility and make their behaviors explicit. We illustrate our method with an immersive driving simulator experiment.
\end{abstract}

\section{INTRODUCTION}

The validation of the credibility and realism of agents in multi-agent simulations is a complex task that has given rise to a lot of work in the domain of multi-agent simulation (see e.g. (Caillou and GilQuijano, 2012)). When the number of agents increases, Drogoul shows (Drogoul et al., 1995) that the validation of such a simulation requires an evaluation of the system at the macroscopic level. However, this does not guarantee validity at the microscopic level, i.e. the validity of the behavior of each agent in the system. In some simulations such as virtual reality environments, where humans coexist with simulated agents, the human point of view is purely local and behavior is considered at the microscopic level. Indeed, if the agents' behavior is inconsistent, user immersion in the virtual environment (i.e. the human's feeling to belong to the virtual environment) is broken (Fontaine, 1992; McGreevy, 1992).

Methods and implementations of behaviors are not directly observable by the user, only the resulting behaviors are. This is why, this notion of credi- bility at the microscopic level does not depend on the way the behaviors are modeled. The outside observer judges them and this perception depends on many factors including sensory elements (visual rendering, haptic, proprioceptive, etc.) (Burkhardt et al., 2003; Stoffregen et al., 2003). The term used in the literature to denote this feeling of realism is called presence effect (Witmer and Singer, 1998). The multiple techniques that are used to enhance the presence effect (called immersion techniques) are mainly evaluated on subjective data. Consequently, the evaluation of the presence effect resulting from a virtual reality $(V R)$ device is done with methods from human sciences.

In this paper, we propose to evaluate the agents' credibility at the microscopic level. To do so, we combine subjective evaluation methods from human sciences with automated behavior traces analysis based on artificial intelligence algorithms. Section 2 presents the state of the art. Section 3 explains the general method we have developed, which relies on data clustering and comparison, and section 4 gives 
the details of the underlying algorithms. Section 5 presents its application on an immersive driving simulator and its results.

\section{STATE OF THE ART}

In this section, we first define the notion of behavior. We then present existing subjective and objective approaches.

\subsection{Levels of Behavior}

Behaviors are a set of observable actions of a person in the environment. There are different levels of human behavior (Pavlov and Anrep, 2003): The lowest level corresponds to simple reflex actions such as going into first gear in a car. These behaviors are similar to the agent's elementary operations. The intermediary level is tactical, it is built on an ordered sequence of elementary behaviors such as a car changing lane on the highway. The highest level of behavior is the strategic level, corresponding to the long term. It is based on a choice of tactics and evolves according to the dynamics of the environment and the mental state of the person (Premack et al., 1978) as in overtaking a truck platoon or choosing a stable cruise speed. In our study, we evaluate the behavior of the agents at the last two levels (tactical and strategic).

\subsection{Subjective Approach}

The subjective approach comes from the $V R$ field and aims at validating the agents' behavior in simulation. It consists in evaluating the general (or detailed) immersion quality via the presence effect using questionnaires (Lessiter et al., 2001). In our case, the notion of presence is too broad because it includes various elements (visual quality, sound quality, etc.) of the device, but does not detail the virtual agents behavior credibility component.

However subparts of the presence effect evaluation are consistent with our goal:

- The behavioral credibility: Users interacting with the agent believe that they observe a human being with his own beliefs, desires and personality (Lester et al., 1997),

- The psychological fidelity: The simulated task generates for the user an activity and psychological processes which are similar to those generated by the real task (Patrick, 1992). The simulator produces a similar behavior to the one required in the real situation (Leplat, 1997).
In this article, we focus on the behavioral credibility and especially on its qualitative and quantitative evaluation. A solution is to set up a mixed system where humans control avatars in the virtual environment. The evaluation of presence or of behavioral credibility is subjective. This is why it is sensitive to psychological phenomena such as the inability to explain one's judgments (Javeau, 1978). Moreover this evaluation does not necessarily explain missing behaviors nor the faults of the behaviors judged as not credible.

That is why we propose to complete these subjective studies with an objective analysis of simulation data.

\subsection{Objective Approach}

The objective approach is generally used in the field of multi-agents systems: It consists in comparing quantitative data produced by humans with data produced by different categories of virtual agents (Caillou and Gil-Quijano, 2012). It aims at verifying that the behavior of the agents is identical to the one observed in reality and therefore at evaluating the realism of the simulation. When the number of agents increases, objective evaluation is generally done at the macroscopic level because real data are both more readily available and easier to compare with simulation outputs (Champion et al., 2002; Maes and Kozierok, 1993).

This macroscopic validation is necessary for the $V R$ but not sufficient to validate the agents' behavior. A valid collective behavior does not imply that the individual behaviors that compose it are valid. Thus, an analysis at the microscopic level is required, although microscopic data analysis and comparison is complex. Some tools are available to summarize interactions of a multi-agent system for manual debugging (Serrano et al., 2012). Nevertheless, as simulation data involving participants consist of more than just message exchange variables, these tools are not directly applicable to complex and noisy data. A solution for data analysis, adopted by (Gonçalves and Rossetti, 2013) for driving tasks consists of classifying participants according to variables. However, our method deals with a larger amount of both variables and participants, increasing the clustering task difficulty. It also provides explicit high-level behaviors via external annotation.

To the best of our knowledge, there is no tool to analyze strategic behavior in simulation combining both a validation of behavioral credibility and similarities between humans and virtual agents. Subjective and objective approaches complement each other 
in two different ways: human expertise and raw data.

\section{OBJECTIVE AND APPROACH}

Our goal is the evaluation of the agents of a multiagents simulation at a microscopic level, in the context of virtual environments. The method we propose is based on the aggregation of individual data (for both agents and human participants) into behavior clusters that will support the actual behavior analysis. In this view, behavior clusters act as abstractions of individual traces. This paper details the computation of such clusters (section 4) and their use for behavior analysis (section 5). The originality of our model is that we use the two available types of data: objective data with logs and subjective data via questionnaires.

\subsection{General Approach}

The general architecture of the method is described in the Figure 1 and the data processing is detailed in Figure 4. It consists of 4 main steps: collection of data in simulation, annotation of this data, automatic clustering of data, and clusters comparison.

The first step of our method is to collect data about human participants. We consider both subjective data, using questionnaires about their general habits and their adopted behaviors in the given task, and objective data, using immersive (or participatory) simulation in the virtual environment. The raw data from participants' experiments in the simulator is called $\log s$ and the answers to questionnaires is called habits in the figure.

The second step is to refine this data by 1) producing new simulations (or "replays") in which the human participant has been replaced by a virtual agent; and 2) having all replays (with human participants and with virtual agents) being annotated by a different set of participants, using the behavior questionnaire. This step produces a set of annotations.

Our objective is twofold. First, we want to study the correlation between participants' categories and their behaviors observed in the simulation so as to verify that the automated clustering of observation data is related to task-related high-level behavior. Second, we need to compare participants' behavior and agents' behavior so as to report on the capability of agents to simulate human behavior. In both cases, this cannot be done on raw data (should it be questionnaires or data logs). Logs, especially in the case of participants, are noisy: two different logs can represent the same type of tactic or strategic behavior.
This is the reason why, in order to generalize the analysis of our logs to a higher behavior level, we propose to use behavior categories (called abstractions in the figure). These categories serve as abstraction to the logs by gathering together, within the same cluster, different logs representative of the same high level behavior. This is done using automatic unsupervised clustering methods (because supervised algorithms require labeling by an expert of a large amount of logs). In the same way, we use clustering methods on the two questionnaires habits and annotations.

The comparison of these abstractions is our final step. We both evaluate the similarity between agents and humans logs and the annotated behaviors (dashed arrow number 1); and between the logs and self-reported habits for humans (dashed arrow number 2). While the first comparison allows us to evaluate the level of credibility of our virtual agents in the simulation, the second one is used to verify that the logs automated clustering corresponds to task-related high-level behaviors. If there is a strong similarity between the composition of behavior clusters and participant self-reported categories (habits), it then means that behavior clusters are meaningful in terms of participant typology. Note that this comparison is meaningful if and only if we use the same sort of indicators for habits and annotations.

\section{Human Participants and Agents}

For the comparison between participants' behavior clusters and agents' ones, we collect the same logs for simulated agents as for the participants. As will be discussed in section 4.2 , the clustering algorithm does not work directly on raw data: we use higherlevel representation based on expert knowledge on the field.

Different types of agents are generated by exploring the parameter space such as normativity, experience, decision parameters ... The agents are placed in an identical situation to that presented to participants, so that the same logs are collected. The clustering is done on both agents and human participants logs, gathered together in the general term of main actors (see section 4.2).

For the evaluation step, it is possible to distinguish three cluster types:

1. The clusters containing both human and agent main actors; they corresponds to high-level behaviors that are correctly reproduced by the agents.

2. Those consisting of simulated agents only; they correspond to behaviors that were produced only by the agents. In most cases, it reflects simula- 


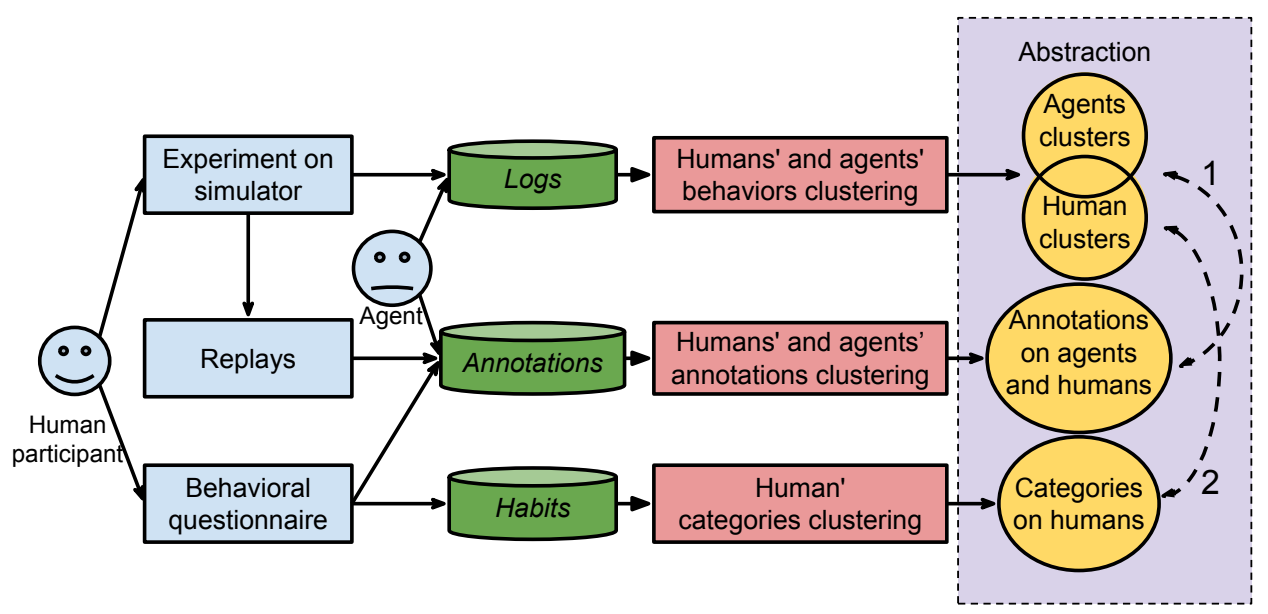

Figure 1: An architecture for behavior analysis and evaluation.

tion errors, but it can also be due to a too small participants sample.

3. Those consisting of participants only; they correspond to behaviors that have not been replicated by the agents, and are thus either lacks in the agent's model, or due to a too small agents sample in the parameter space.

In the end, we combine this agent-human comparison with the annotation-habits analysis: The participants' behavior clusters are correlated to their habits categories. Furthermore, the composition of the behavior clusters in term of simulated agents and participants allows us to give explicit information about those agents.

\subsection{Case Study}

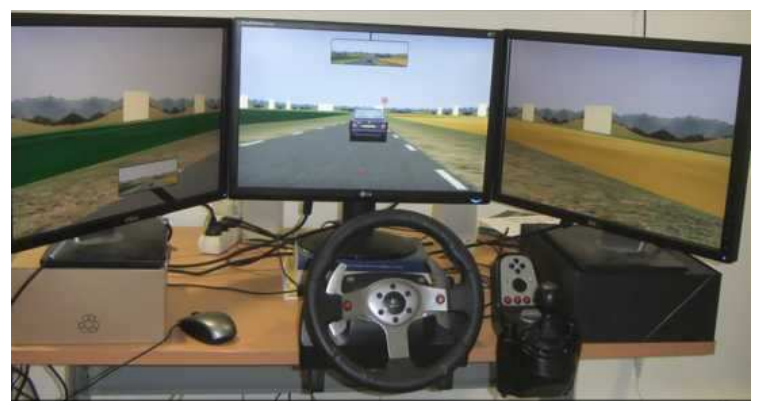

Figure 2: Driving simulator device with 3 screens, steering wheel, gearbox and a set of pedals.

Our method was tested in the context of driving simulators. We want to evaluate the realism and credibility of the behavior of the IFSTTAR's road traffic simulator's agents (see Figure 2) by using the ARCHISIM driving simulator (Champion et al.,
2001). To do this, the participants drive a car on a road containing simulated vehicles. The circuit (shown in Figure 3) provides a driving situation on a single carriage way with two lanes in the same direction. It corresponds to about 1 minute of driving. The main actor encounters a vehicle at low speed on the right lane.

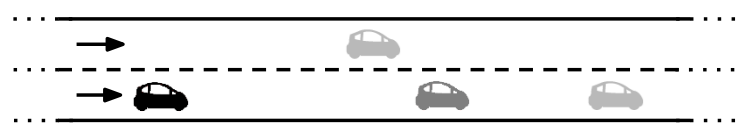

Figure 3: Scenario: The main actor (in black) is driving on a single carriage way with two lanes in the same direction with a smooth traffic flow. Then, a vehicle at low speed on the right lane (in dark gray) disturbs the traffic.

Our method is illustrated in the following sections with this application to the study of driving behavior. However, the presented method may be used in any kind of participatory simulation, by choosing relevant task-related questionnaires.

\section{DATA PROCESSING METHOD}

In this section, we detail the different elements of our behavioral validation method and the algorithms that we use.

\subsection{Clustering of Main Actors Categories}

We first describe the habits questionnaire and the annotations questionnaire applied to the driving simulation, and then detail the clustering algorithm. 


\subsubsection{Participants Habits}

In the first place it is necessary to submit a behavior questionnaire specific to the field before the experiment to characterize the general behavior of the participant in the studied activity. In the context of our application to driving simulators, we chose the Driving Behavior Questionnaire $(D B Q)$ (Reason et al., 1990). It provides a general score, but also scores on 5 subscales: 1) slips; 2) lapses; 3) mistakes; 4) unintended violations; and 5) deliberate violations. In addition, it supplies 3 subscales related to the accident risk: 1) no risk; 2) possible risk; and 3) definite risk.

\subsubsection{Annotation of Main Actors Behaviors}

An adopted behavior in a precise situation may not correspond to the participant's general behavior. For example, in driving simulators, the general driving behavior captured by the $D B Q$ may not correspond to the participant's behavior in the precise studied situation. Furthermore, the general behavior questionnaire is completed by the driver himself about his own behaviors. This adds a bias due to introspection.

This is why we need to use a second questionnaire called annotations. This questionnaire is completed by a different set of participants. It avoids the introspection bias. Furthermore, having a population which is observing the situation allows us to collect situation specific information. The questions are rated on a Likert-type scale (Likert, 1932). In our application to driving simulators, the questionnaire contains a question rated on a 7 points scale (and no opinion) from no to yes for each of the $5 D B Q$ subscales.

The 3 risk-related subscales are merged in a unique question named accident risk rated on a 3 points scale (and no opinion). We also add a question related to the perceived control on the same 7 points scale with the purpose of evaluating the main actors control in general. At last, a question asking if the main actor is a human or a simulated agent is added in order to compare how the behavior clustering and the annotators separated the participants from the agents.

\subsubsection{From Data to Categories}

In the general case (independently from the application domain), using behavior questionnaires, we obtain qualitative data on Likert-type scales. The answers are transformed into quantitative data via a linear numeric scale. Scale scores of questionnaires are then calculated by adding the scale-related questions, and normalized between 0 and 1 . Once data are processed, we classify the participants' scores using a clustering algorithm to obtain drivers categories. This allows us to obtain clusters corresponding to participants' habits and how they are annotated. As seen in section 3.1, the algorithm must be unsupervised with a free number of clusters. Several algorithms exist in the literature to this purpose, such as Cascade $K$ means with the Variance Ratio Criterion (CalinskiHarabasz) (Caliński and Harabasz, 1974), X-means based on the Bayesian Information Criterion (Pelleg et al., 2000), or Self-Organizing Maps (Kohonen, 1990).

We chose to use the Cascade K-means algorithm which executes several $K$-means for $K \in\{1, \ldots, N\}$. The classic $K$-means algorithm uses $K$ random initial centroids. It then proceeds those two steps alternatively until convergence: 1) The assignment step which assigns each main actor $m a$ to the cluster $C_{i}$ whose mean yields the least within-cluster sum of squares $m_{i}$ at time $t$ (see Equation (1));2) The update step which calculates the new means $m$ to be the centroids of the main actors in the new clusters at time $t+1$ (see Equation (2)).

$$
\begin{gathered}
\forall j \in\{1, \ldots, k\} \\
C_{i}^{(t)}=\left\{m a_{p}:\left\|m a_{p}-m_{i}^{(t)}\right\|^{2} \leq\left\|m a_{p}-m_{j}^{(t)}\right\|^{2}\right\} \\
m_{i}^{(t+1)}=\frac{1}{\left|C_{i}^{(t)}\right|} \sum_{m a_{j} \in C_{i}^{(t)}} m a_{j}
\end{gathered}
$$

The initialization of the clusters is done with $K$ means ++ (Arthur and Vassilvitskii, 2007) which allows a better distribution of clusters' centers in accordance with the data. To do so, the centroid of the first cluster is initialized randomly among the main actors. Until having $K$ clusters, the algorithm computes the distance of each main actor to the last selected centroid. Then, it selects the centroid of a new cluster among the main actors. The selection is done randomly according to a weighted probability distribution proportional to their squared distance.

Finally, we must select the "best" number of clusters with respect to our clustering goal. This is done using the Variance Ratio Criterion which takes into account the inter-distance (i.e. the within-cluster error sum of squares $W$ ) and intra-distance (i.e. the between-cluster error sum of squares $B$ ) of the clusters (Caliński and Harabasz, 1974). Let $\left|C_{k}\right|$ be the number of elements in the cluster $C_{k}, \overline{C_{k}}$ be the barycenter of this cluster and $\overline{\mathbb{C}}$ be the barycenter of all main actors (i.e. the clustering). Then, the Variance Ratio Criterion $\mathrm{CH}$ for $\mathrm{K}$ clusters is as described bellow (in Equation (3)): 


$$
\begin{gathered}
C H(K)=\frac{B /(K-1)}{W /(N-K)} \\
B=\sum_{k=1}^{K}\left|C_{k}\right|\left\|\overline{C_{k}}-\overline{\mathbb{C}}\right\|^{2} \\
W=\sum_{k=1}^{K} \sum_{n=1}^{N}\left\|m a_{k, n}-\overline{C_{k}}\right\|^{2}
\end{gathered}
$$

\subsection{Clustering of Behaviors}

This section describes how raw data logs are turned into clusters, within a series of pre-processing and clustering methods. The figure 4 shows the preprocessing applied to the logs in order to obtain clusters. Squares indicate the data name and its shape with the number of variables $(X)$, the number of indicators $(K)$, the number of main actors $(N)$, and the time $(T)$. The used algorithms are in squircles above arrows. The section 4.2.1 (on the top of the figure) describes the logs of the main actors; the section 4.2.2 (on the middle of the figure) explains the pre-processing; and the section 4.2.3 (on the right of the figure) explains the clustering algorithm.

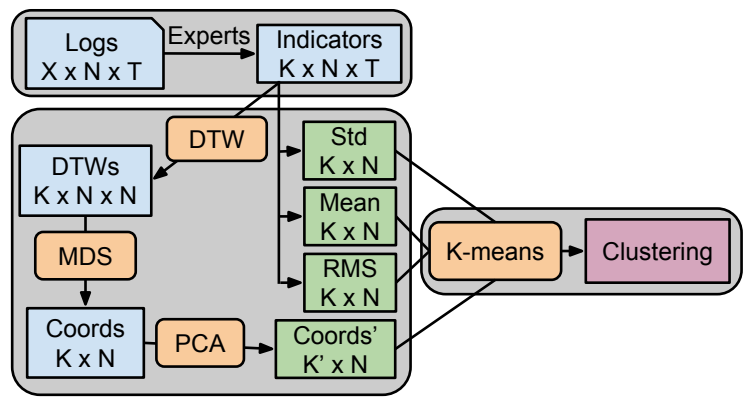

Figure 4: Logs pre-processing and clustering.

\subsubsection{Main Actors Logs}

During the simulation we collect the logs of the main actor (participant or agent), of neighboring agents and of the environment. These logs are then used for the clustering of tactical and strategic behaviors. The data to be recorded must be defined by experts in the domain of application.

In our traffic simulation example, we collect each $300 \mathrm{~ms}$ from 8 to 13 variables. The variables shared by all the main actors are the time, the milepost, the road, the gap and the cap to the lane axis, the speed, the acceleration, and the topology. Specific variables to the driven vehicles are added: the wheel angle, the pressure on pedals (acceleration, brake and clutch), and the gear.
The road traffic experts chose the following indicators: some high-level variables like the intervehicles distance and time, the jerk (the derivative of acceleration with respect to time), the time to collision (under the assumption of constant speeds for both vehicles), and the number of lane changings (which is not a temporal indicator) ; as well as some low-level variables such as speed, acceleration, and lateral distance to the road axis.

\subsubsection{Pre-processing}

Some significant indicators dependent on the application field cannot be directly obtained. For this reason, field experts are consulted to identify important indicators. Then we calculate the indicators from the logs for those that could not be collected.

In the context of a dynamic simulation, most of the indicators are temporal. The data to classify are thus ordered sequences of values for each main actor. In order to classify those data, two ways exist: to use an algorithm taking temporal data as input or to use flat data by concatenating temporal indicators related to a participant on a single line. The first solution significantly increases the algorithms' complexity because they must take into account the possible temporal offsets of similar behaviors. The second one ignores temporal offsets but permits the application of classic algorithms.

We chose a hybrid solution of data pre-processing which allows us both to have a single set of attributes for each participant and to take into account temporal offsets. To do this, we generate as many vectors as main actors (participants and virtual agents). Each vector contains the following information extracted from the indicators identified by the field experts: $a$ ) mean values; $b$ ) standard deviations; $c$ ) root mean squares; and $d$ ) temporal aggregations. Temporal indicators are compared with an algorithm taking into account temporal offsets.

The adopted solution for the pre-processing of temporal offsets is to use a pattern matching algorithm such as Dynamic Time Warping $(D T W)$ or Longest Common Subsequence ( $L C S$ ). We chose the DTW algorithm which calculates the matching cost between two ordered sequences (i.e. indicators ind $d^{a}$ and $i n d^{b}$ ) in order to measure their similarity (Berndt and Clifford, 1994). Let $T$ be the number of simulation time steps. The algorithm computes a $T \times T$ matrix. It initializes the first row and the first column to infinity, and the first element to 0 . It then computes each elements of the matrix $M_{i, j} \forall(i, j) \in\{2, \ldots, T+1\}^{2}$ according to the distance between the two sequences at this time $t$ and to the matrix element neighborhood (see Equation (4)). As $D T W$ complexity is $O\left(N^{2}\right)$, we 
use an approximation of this algorithm: the FastDTW algorithm which has order of $O(N)$ time and memory complexity (Salvador and Chan, 2007).

$$
\begin{gathered}
D T W[i, j] \leftarrow \operatorname{distance}\left(\text { ind }_{i}^{a}, \text { ind }_{j}^{b}\right)+ \\
\min (\operatorname{DTW}[i-1, j], D T W[i, j-1], D T W[i-1, j-1])
\end{gathered}
$$

As DTW calculates the similarity between two instances of a temporal variable, the less the instances are similar, the more the cost increases. Let inds be the set of indicators and $K=\mid$ ind $s \mid$ be the number of indicators. For each indicator ind $\in$ inds, we calculate the $N \times N$ mutual distances matrix $D_{D T W}^{\text {ind }}$, where $N$ is the number of main actors (participants and agents).

In order to include $D T W$ similarities as new variables describing the main actors, we use a MultiDimensional Scaling algorithm $(M D S)$ to place each main actor in a dimensional space. The algorithm assigns a point to each instance in a multidimensional space and tries to minimize the number of space dimensions. The goal is to find $N$ vectors $\left(\operatorname{coord}_{1}, \ldots, \operatorname{coord}_{N}\right) \in \mathbb{R}^{N}$ such that $\forall(i, j) \in$ $N^{2},\left\|\operatorname{coord}_{i}-\operatorname{coord}_{j}\right\| \approx D_{D T W}^{\text {ind }}(i, j)$.

As $D T W$ is a mathematical distance, the $M D S$ algorithm applied to each $D_{D T W}$ is able to minimize the number of space dimensions to 1 (i.e. a vector of coordinates). Then we have as many vectors of coordinates as indicators.

Indicators' coordinates may be correlated among each others but the $K$-means algorithm uses a dimensional space of which the axes are orthogonal to each other. In order to apply this algorithm, we need to project the data on an orthogonal hyperplane of which the axes are two by two non-correlated.

The Principal Component Analysis (PCA) calculates the non-correlated axes which give a maximal dispersion of the data. It is then possible to reduce the number of dimensions avoiding redundant information by compressing them. Data are represented in a matrix of coordinates $C$ with $K$ random variables $\left\{\right.$ ind $_{1}, \ldots$, ind $\left._{K}\right\}$ containing $N$ independent realizations. This matrix is standardized according to the center of gravity $\left(\overline{\text { ind }_{1}}, \ldots, \overline{\text { ind }_{K}}\right)$ (with $\overline{\text { ind }}$ the arithmetic mean) and to the standard deviation $\sigma$ of the random variables. It is then possible to calculate the correlation matrix: $\frac{1}{N} \cdot \widetilde{C}^{T} \cdot \widetilde{C}$. The $P C A$ looks for the axis $u$ which maximizes the variance of the data. To do so, it calculates a linear combination of the random variables in order to project the data on this axis: $\pi_{u}(C)=C \cdot u$. We keep the same number of axes $K^{\prime}$ for the projected indicators as for the indicators $(K)$.

$$
\widetilde{C}=\left[\begin{array}{ccc}
\frac{i n d_{1,1}-\overline{\text { ind }}}{\sigma\left(i n d_{1}\right)} & \ldots & \frac{i n d_{1, K}-\overline{\text { ind }_{K}}}{\sigma\left(i n d_{K}\right)} \\
\vdots & \ddots & \vdots \\
\frac{i n d_{N, 1}-\overline{\text { ind }}}{\sigma\left(i n d_{1}\right)} & \cdots & \frac{i n d_{N, K}-\overline{\text { ind }}}{\sigma\left(i n d_{K}\right)}
\end{array}\right]
$$

\subsubsection{Behavior Clusters}

Finally, we apply on the $P C A$ projected indicators the same $K$-means algorithm as the one applied on the questionnaire's scores in order to classify these data (normalized between 0 and 1 ). We thus obtain behavior clusters of main actors, as shown in Figure 4.

\subsection{Clusterings Comparison}

Now that we have annotations clustering, behaviors clustering on main actors and habits clustering on participants, we want to compare the clusters composition between the annotations and the behaviors, and between the habits and the behaviors.

As we want to compare clusterings, we need a similarity measure between two clusterings $\mathbb{C}_{1}$ and $\mathbb{C}_{2}$. We use the Adjusted Rand Index (ARI) (Hubert and Arabie, 1985) - a well known index recommended in (Milligan and Cooper, 1986) - which is based on pair counting: a) $N_{00}$ : the number of pairs that are in the same set in both clusterings (agreement); b) $N_{11}$ : the number of pairs that are in different sets in both clusterings (agreement); and $c$ ) $N_{01}$ and $N_{10}$ : the number of pairs that are in the same set in one clustering and in different sets in the other (disagreement) and vice-versa. The Rand Index $R I \in[0,1]$ is described in Equation (6) (Rand, 1971). The Adjusted Rand Index $A R I \in[-1,1]$ is calculated using a contingency table $\left[n_{i j}\right]$ where $n_{i j}$ is the number of agreements between instances $i$ and $j: n_{i j}=\left|\mathbb{C}_{1}^{i} \cap \mathbb{C}_{2}^{j}\right|$ (see Equation (7)). It is a corrected-for-randomness version of the $R I$ : Where the expected value of $R I$ for two random clusterings is not constant, the expected value of $A R I$ is 0 .

$$
\begin{gathered}
R I\left(\mathbb{C}_{1}, \mathbb{C}_{2}\right)=\frac{N_{00}+N_{11}}{N_{00}+N_{11}+N_{01}+N_{10}} \\
\operatorname{ARI}\left(\mathbb{C}_{1}, \mathbb{C}_{2}\right)=\frac{R I\left(\mathbb{C}_{1}, \mathbb{C}_{2}\right)-E\left[R I\left(\mathbb{C}_{1}, \mathbb{C}_{2}\right)\right]}{1-E\left[R I\left(\mathbb{C}_{1}, \mathbb{C}_{2}\right)\right]}
\end{gathered}
$$

where

$$
E\left[R I\left(\mathbb{C}_{1}, \mathbb{C}_{2}\right)\right]=\left[\sum_{i}\left(\begin{array}{c}
\sum_{k} n_{i k} \\
2
\end{array}\right) \sum_{j}\left(\begin{array}{c}
\sum_{l} n_{l j} \\
2
\end{array}\right)\right] /\left(\begin{array}{l}
n \\
2
\end{array}\right)
$$




\section{EXPERIMENTATION}

The participants to our driving simulation experiment are regular drivers aged from 24 to 59 (44\% female). Our experiment is carried out on a device comprising a steering wheel, a set of pedals, a gearbox and 3 screens allowing sufficient lateral field of view (see Figure 2). These screens are also used to integrate the rear-view mirror and the left-hand mirror. 23 participants used this device.

Firstly, the Driver Behavior Questionnaire is submitted before the simulation. Secondly, a first test without simulated traffic is performed for the participant to get accustomed to the functioning of the simulator and to the circuit. Then, the participant performs the scenario, this time in interaction with simulated vehicles. It should be noted that as the behavior of simulated vehicles is not scripted, situations differ more or less depending on the main actor behavior. The data are then recorded for the processing phase. A video is also made for the replay. Finally, another population of 6 participants fills the annotations questionnaire after viewing the video replay of the simulation in order to evaluate the adopted behaviors of the main actors (i.e. 23 participants and 14 agents).

One participant had simulator sickness but was able to finish the experiment, and one annotator had dizziness and ceased watching.

\section{$5.1 \quad$ Results}

We have compared the habits clustering, the annotations clustering, and the behaviors clustering. The composition of the clusterings is illustrated with three graphs. Agents are represented with rectangles and are named $a \#$. Participants are represented with ellipses and are named $s \#$. The main actors of one clustering are grouped together within rectangles containing the cluster number on the top. The others clustering's main actors are grouped together by color and the cluster number is written just bellow the main actors names.

\subsubsection{Habits \& Annotations Clusters}

We want to compare the $D B Q$ scales and the summarized $D B Q$ scales of our annotations questionnaire. The figure 5 shows the habits clustering (within rectangles) and the annotations clustering (grouped together by color), and their similarity. As the habits clustering from the $D B Q$ questionnaire is only filled by simulation participants, we do not display the agents from the annotations clustering. The habits clustering contains 3 clusters which are close to the 2
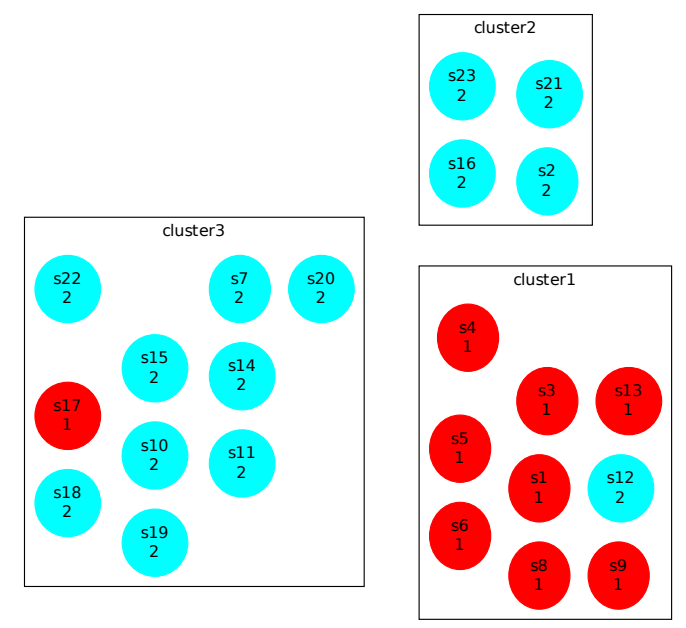

Figure 5: Comparison of participants between habits clustering (within rectangles) and annotations clustering (grouped together by color).

clusters of the annotations clustering. clusterl contains nearly all participants of the cluster (1) (excepted $s 12$ ). cluster 2 is composed of cluster (2) participants only. Also, cluster 3 is mainly composed of cluster (2) participants (excepted $s 17$ ). The rand index is 0.71 and the adjusted rand index is 0.42 . This means that our summarized $D B Q$ scales in the annotations questionnaire is meaningful with regard to driver behavior habits.

\subsubsection{Behavior Clusters \& Annotations Clusters}

With the behavior clustering on main actors, we are able to analyze how many human behaviors are reproduced by the agents, how many human behaviors are not adopted by the agents, and how many agent behaviors are not adopted by participants. We are also capable of making explicit those behaviors via the similarity with annotations clusters if relevant.

The figure 6 presents the behaviors clustering (within rectangles) and the annotations ${ }^{1}$ clustering grouped together by color. The number of clusters is similar in both clusterings ( 3 behaviors versus 2 types of annotations). The rand index is 0.59 and the adjusted rand index is 0.17 .

- clusterl contains one participant and nearly all the agents (excepted a5). Most of its main actors are annotated in the same way (i.e. in cluster (1)). So, the main actors of the cluster 1 adopted a similar driving behavior and were annotated in

\footnotetext{
1 except for the human or simulated agent question which is not related to the adopted behavior.
} 


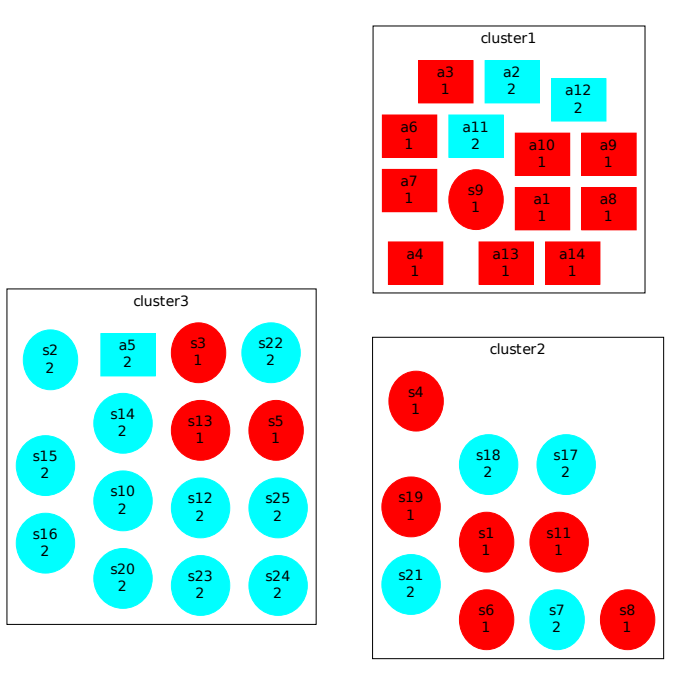

Figure 6: Comparison of main actors between behavior clustering within rectangles and annotations clustering grouped together by color.

the same way, i.e.: the highest score on perceived control question and the lowest scores on the other questions. Therefore, they are judged as careful drivers.

- cluster 2 is only composed of participants which are mixed between the two annotations clusters.

- cluster3 is mainly composed of participants (and the agent $a 5$ ). Those participants are largely annotated in the same cluster (2), which has the lowest score on the perceived control question and the highest scores on other questions, meaning that they are judged as unsafe drivers.

\subsubsection{Behavior Clusters \& Habits}

We have compared the drivers habits using the $D B Q$ questionnaire with the adopted behavior. As the habits clustering from the $D B Q$ questionnaire is only filled by simulation participants, we do not displayed the agents from the annotations clustering. The Variance Ratio Criterion selects 3 clusters. The rand index is low (0.48) as is the adjusted rand index (0.07). The clustering contains a singleton cluster and two other clusters, each containing a mixture of all the $D B Q$ clusters, meaning that the behavior clustering does not correspond to the habits clustering. It validates the use of annotations by observers, which are closer to data clustering results.

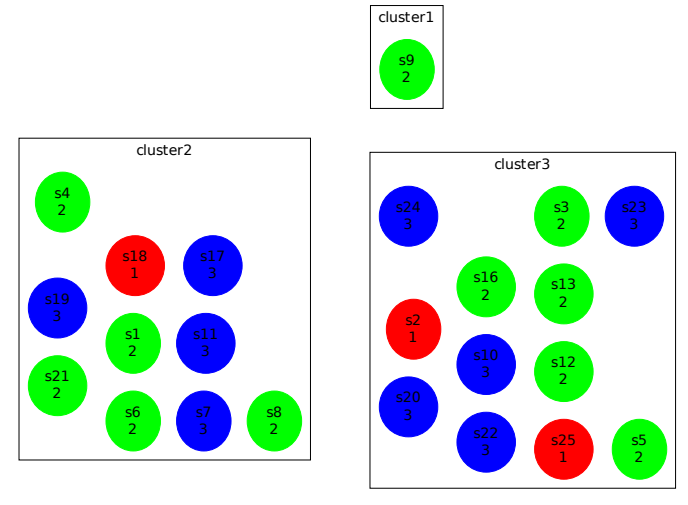

Figure 7: Comparison of main actors between behavior clustering within rectangles and habits clustering grouped together by color.

\subsection{Discussion}

Firstly, there is no significant similarity between habits clustering and behaviors clustering. This might be due to the general approach of the $D B Q$ questionnaire. The driver's habits may differ from the adopted behavior. The $D B Q$ is filled by the driver itself. The introspection bias may be the reason of the differences. This is also an issue for us because we cannot apply it to the agent. The 3 scales dealing with the risk could be another problem: $8 \%$ of the participants had some high scores on the No risk scale and the Definite risk scale but a low score one the Possible risk scale. Another problem is that the same type of participant in term of $D B Q$ cluster can adopt different behaviors for the same situation, leading to different logs. Similarly, the same behavior can be adopted by different types of participants. This is an issue to analyze the similarity between the habits clustering and the behaviors clustering with the ARI measure. A solution could be to merge the subsets for which all main actors are also in the same cluster in the other clustering.

Secondly, we have a significant similarity between annotations and behavior clusterings, meaning that we are able to classify our logs data into high-level behavior clusters which are meaningful in term of driving annotations. Nevertheless the two clusterings are not identical with regard to the clusters composition nor with regard to the clusters number. This could be due to the few number of annotators, we are currently increasing this population. Furthermore, the behavior clustering is done on noisy indicators for human participants and on smooth indicators for agents. A solution might be to add a noise on the agents data or to smooth the participants data. This problem may come from the clustering algorithm which is a classic 
but basic one. We have to test with advanced algorithms like $E M$ or a temporal algorithm.

In the comparison of annotations and behaviors, one agent was in a mainly human composed cluster. Does this mean that it is able to simulate the majority of driver's behaviors of this cluster which is cautious ? If it is, we can then consider that this cautious behavior is an agents ability. To verify this assumption, we would need a specific test in which the parameter set that was used for the agent $a 5$ is confronted to different situations, and compared with logs and annotations of cautious human drivers. Conversely, one participant was in the mainly agent composed cluster judged as dangerous for a majority of them. This requires further study to understand what was specific in this subject's driving behavior that was similar to the agents' behaviors. cluster 2 does not contain any agent, meaning that the agent's model is not able to reproduce this human driving behavior (i.e. this behavior is lacking in the agent's model). Another type of cluster - which does not appear in this experiment is composed of agents only. In that case, we can consider - as no participant adopted this behavior - that the agents behavior is inaccurate (i.e. is an error) and should be investigated further.

\section{CONCLUSION \& PERSPECTIVES}

This paper presents a method to study the agents' behavioral credibility through an experiment in a virtual environment. This validation is original in coupling a subjective analysis of the agents' behavioral credibility (via human sciences questionnaires and annotations) with an objective analysis of the agents' abilities. This analysis is based on behaviors clustering which allows us to obtain behaviors categories at a higher level than raw data. The method is generic for mixed simulation where agents and humans interact. When applied to a new domain, some of the tools have to be adapted, such as the choice of the behavior questionnaire which is domain-specific. The method is fully implemented, built on the Weka toolkit. The software shall be made available in the future.

Our validation method was applied to the road traffic simulation. This experiment showed that the methodology is usable for mixed and complex VEs and that it is possible to obtain high-level behaviors from the logs via our abstraction. A larger annotators population should provide more evidence of the method's robustness.

Several tracks for further work remain to explore. On the clustering part, the evaluation of multiple algo- rithms should enable to better assess their relevance. To do so, the use of the results of the comparison between annotations clusters and observed behavior clusters allows us to choose the most pertinent algorithm depending on the application. Another research open issue - as annotation are similar to behaviors whereas habits differ - is how the behaviors clustering evolve through multiple situations of a longer scenario, whether the participants clusters remain stable or change in number or composition.

\section{REFERENCES}

Arthur, D. and Vassilvitskii, S. (2007). k-means++: The advantages of careful seeding. In Proceedings of the eighteenth annual ACM-SIAM symposium on Discrete algorithms, pages 1027-1035. Society for Industrial and Applied Mathematics.

Berndt, D. and Clifford, J. (1994). Using dynamic time warping to find patterns in time series. In $K D D$ workshop, volume 10, pages 359-370.

Burkhardt, J. M., Bardy, B., and Lourdeaux, D. (2003). Immersion, réalisme et présence dans la conception et l'évaluation des environnements virtuels. Psychologie française, 48(2):35-42.

Caillou, P. and Gil-Quijano, J. (2012). Simanalyzer: Automated description of groups dynamics in agent-based simulations. In Proceedings of the 11th International Conference on Autonomous Agents and Multiagent Systems-Volume 3, pages 1353-1354. International Foundation for Autonomous Agents and Multiagent Systems.

Caliński, T. and Harabasz, J. (1974). A dendrite method for cluster analysis. Communications in Statistics-theory and Methods, 3(1):1-27.

Champion, A., Éspié, S., and Auberlet, J. M. (2001). Behavioral road traffic simulation with archisim. In Summer Computer Simulation Conference, pages 359364. Society for Computer Simulation International; 1998.

Champion, A., Zhang, M. Y., Auberlet, J. M., and Espié, S. (2002). Behavioral simulation: Towards high-density network traffic studies. ASCE.

Drogoul, A., Corbara, B., and Fresneau, D. (1995). Manta: New experimental results on the emergence of (artificial) ant societies. Artificial Societies: the computer simulation of social life, pages 190-211.

Fontaine, G. (1992). The experience of a sense of presence in intercultural and international encounters. Presence: Teleoperators and Virtual Environments, 1(4):482-490.

Gonçalves, J. and Rossetti, R. J. F. (2013). Extending sumo to support tailored driving styles. 1st SUMO User Conference, DLR, Berlin Adlershof, Germany, 21:205-211.

Hubert, L. and Arabie, P. (1985). Comparing partitions. Journal of classification, 2(1):193-218. 
Javeau, C. (1978). L'enquête par questionnaire: manuel à l'usage du praticien. Editions de l'Université de Bruxelles.

Kohonen, T. (1990). The self-organizing map. Proceedings of the IEEE, 78(9):1464-1480.

Leplat, J. (1997). Simulation et simulateur: principes et usages. Regards sur l'activité en situation de travail: contribution à la psychologie ergonomique, pages 157-181.

Lessiter, J., Freeman, J., Keogh, E., and Davidoff, J. (2001). A cross-media presence questionnaire: The itc-sense of presence inventory. Presence: Teleoperators \& Virtual Environments, 10(3):282-297.

Lester, J. C., Converse, S. A., et al. (1997). The persona effect: affective impact of animated pedagogical agents. In Proceedings of the SIGCHI conference on Human factors in computing systems, pages 359-366. ACM.

Likert, R. (1932). A technique for the measurement of attitudes. Archives of psychology.

Maes, P. and Kozierok, R. (1993). Learning interface agents. In Proceedings of the National Conference on Artificial Intelligence, pages 459-459. John Wiley \& Sons LTD.

McGreevy, M. W. (1992). The presence of field geologists in mars-like terrain. Presence: Teleoperators and Virtual Environments, 1(4):375-403.

Milligan, G. W. and Cooper, M. C. (1986). A study of the comparability of external criteria for hierarchical cluster analysis. Multivariate Behavioral Research, 21(4):441-458.

Patrick, J. (1992). Training: Research and practice. Academic Press.

Pavlov, I. P. and Anrep, G. V. (2003). Conditioned reflexes. Dover Pubns.

Pelleg, D., Moore, A., et al. (2000). X-means: Extending k-means with efficient estimation of the number of clusters. In Proceedings of the Seventeenth International Conference on Machine Learning, volume 1, pages 727-734. San Francisco.

Premack, D., Woodruff, G., et al. (1978). Does the chimpanzee have a theory of mind. Behavioral and brain sciences, 1(4):515-526.

Rand, W. M. (1971). Objective criteria for the evaluation of clustering methods. Journal of the American Statistical association, 66(336):846-850.

Reason, J., Manstead, A., Stradling, S., Baxter, J., and Campbell, K. (1990). Errors and violations on the roads: a real distinction? Ergonomics, 33(1011):1315-1332.

Salvador, S. and Chan, P. (2007). Toward accurate dynamic time warping in linear time and space. Intelligent Data Analysis, 11(5):561-580.

Serrano, E., Muñoz, A., and Botia, J. (2012). An approach to debug interactions in multi-agent system software tests. Information Sciences, 205:38-57.

Stoffregen, T. A., Bardy, B. G., Smart, L. J., and Pagulayan, R. J. (2003). On the nature and evaluation of fidelity in virtual environments. Virtual and adaptive environments: Applications, implications, and human performance issues, pages 111-128.
Witmer, B. G. and Singer, M. J. (1998). Measuring presence in virtual environments: A presence questionnaire. Presence, 7(3):225-240. 